\title{
Can Corpus Based Measures be Used for Comparative Study of Languages?
}

\author{
Anil Kumar Singh \\ Language Tech. Research Centre \\ Int'1 Inst. of Information Tech. \\ Hyderabad, India \\ anileresearch.iit.net
}

\author{
Harshit Surana \\ Language Tech. Research Centre \\ Int'l Inst. of Information Tech. \\ Hyderabad, India \\ surana.h@gmail.com
}

\begin{abstract}
Quantitative measurement of inter-language distance is a useful technique for studying diachronic and synchronic relations between languages. Such measures have been used successfully for purposes like deriving language taxonomies and language reconstruction, but they have mostly been applied to handcrafted word lists. Can we instead use corpus based measures for comparative study of languages? In this paper we try to answer this question. We use three corpus based measures and present the results obtained from them and show how these results relate to linguistic and historical knowledge. We argue that the answer is yes and that such studies can provide or validate linguistic and computational insights.
\end{abstract}

\section{Introduction}

Crosslingual and multilingual processing is acquiring importance in the computational linguistics community. As a result, semi-automatic crosslingual comparison of languages is also becoming a fruitful area of study. Among the fundamental tools for crosslingual comparison are measures of inter-language distances. In linguistics, the study of inter-language distances, especially for language classification, has a long history (Swadesh, 1952; Ellison and Kirby, 2006). Basically, the work on this problem has been along linguistic, archaeological and computational streams. Like in other disciplines, computational methods are in- creasingly being combined with other more conventional approaches (Dyen et al., 1992; Nerbonne and Heeringa, 1997; Kondrak, 2002; Ellison and Kirby, 2006). The work being presented in this paper belongs to the computational stream.

Even in the computational stream, most of the previous work on inter-language distances had a strong linguistic dimension. For example, most of the quantitative measures of inter-language distance have been applied on handcrafted word lists (Swadesh, 1952; Dyen et al., 1992). However, with increasing use of computational techniques and the availability of electronic data, a natural question arises: Can languages be linguistically compared based on word lists extracted from corpora. A natural counter-question is whether such comparison will be valid from linguistic and psycholinguistic points of view. The aim of this paper is to examine such questions.

To calculate inter-language distances on the basis of words in corpora, we propose two corpus based distance measures. They internally use a more linguistically grounded distance measure for comparing strings. We also present the results obtained with one purely statistical measure, just to show that even naive corpus based measures can be useful. The main contribution is to show that even noisy corpora can be used for comparative study of languages. Different measures can give different kinds of insights.

\section{Related Work}

Typology or history of languages can be studied using spoken data or text. There has been work on the former (Remmel, 1980; Kondrak, 2002), but we 
will focus only on text. An example of a major work on text based similarity is the paper by Kondrak and Sherif (Kondrak and Sherif, 2006). They have evaluated various phonetic similarity algorithms for aligning cognates. They found that learning based algorithms outperform manually constructed schemes, but only when large training data is used.

A recent work on applications of such techniques for linguistic study is by Heeringa et al. (Heeringa et al., 2006). They performed a study on different variations of string distance algorithms for dialectology and concluded that order sensitivity is important while scaling with length is not. It may be noted that Ellison and Kirby (Ellison and Kirby, 2006) have shown that scaling by distance does give significantly better results. Nakleh et al. (Nakleh et al., 2005) have written about using phylogenetic techniques in historical linguistics as mentioned by Nerbonne (Nerbonne, 2005) in the review of the book titled 'Language Classification by Numbers' by McMahon and McMahon (McMahon and McMahon, 2005). All these works are about using quantitative techniques for language typology and classification etc.

\section{Inter-Language Comparison}

Inter-language comparison is more general than measuring inter-language distance. In addition to the overall linguistic distance, the comparison can be of more specific characteristics like the proportion of cognates derived vertically and horizontally. Or it can be of specific phonetic features (Nerbonne, 2005; McMahon and McMahon, 2005). Quantitative measures for comparing languages can first be classified according to the form of data being compared, i.e., speech, written text or electronic text. Assuming that the text is in electronic form, the most common measures are based on word lists. These lists are usually prepared by linguists and they are often in some special notation, e.g. more or less a phonetic transcription.

The measures can be based on inter-lingual or on intra-lingual comparison of phonetic forms (Ellison and Kirby, 2006). They may or may not use statistical techniques like measures of distributional similarity (cross entropy, KL-divergence, etc.). These characteristics of measures may imply some linguis- tic or psycholinguistic assumptions. One of these is about a common phonetic space.

\section{Common Phonetic Space}

Language distance can be calculated through crosslingual as well as intra-lingual comparison. Many earlier attempts (Nerbonne and Heeringa, 1997; Kondrak, 2002) were based on crosslingual comparison of phonetic forms, but some researchers have argued against the possibility of obtaining meaningful results from crosslingual comparison of phonetic forms. This is related to the idea of a common phonetic space. Port and Leary (Port and Leary, 2005) have argued against it. Ellison and Kirby (Ellison and Kirby, 2006) argue that even if there is a common space, language specific categorization of sound often restructures this space. They conclude that if there is no language-independent common phonetic space with an equally common similarity measure, there can be no principled approach to comparing forms in one language with another. They suggest that language-internal comparison of forms is better and psychologically more well-grounded.

This may be true, but should we really abandon the approach based on crosslingual comparison? As even Ellison and Kirby say, it is possible to argue that there is a common phonetic space. After all, the sounds produced by humans are determined by human physiology. The only matter of debate is whether common phonetic space makes sense from the cognitive point of view. We argue that it does. In psychology, there has been a long debate about a similar problem which can be stated in terms of a common chromatic space. Do humans in different cultures see the same colors? There is still no conclusive answer, but many computational techniques have been tried to solve real world problems like classifying human faces, seemingly with the implicit assumption that there is a common chromatic space. Such techniques have shown some success (sheng Chen and kai Liu, 2003).

Could it be that we are defining the notion of a common chromatic (or phonetic) space too strictly? Or that the way we define it is not relevant for computational techniques? In our view the answer is yes. We will give a simple, not very novel, exam- 
ple. The phoneme $t$ as in the English word battery is not present in many languages of the world. When a Thai speaker can not say battery, with the correct $t$, he will say battery with $t$ as in the French word entre. Such substitution will be very regular. The point is that even if phonetic space is restructured for a particular language, we can still find which segments or sections of two differently structured phonetic spaces are close. Cyan may span different ranges (on the spectrum) in different cultures, but the ranges are likely to be near to one another. Even if some culture has no color which can be called cyan, one or two of the colors that it does have will be closer to cyan than the others. The same is true for all the other colors and also for sounds. If we use fuzzy similarity measures to take care of such differently structured cognitive spaces, cross-lingual comparison may still be meaningful for certain purposes. This argument is in defence of cross-lingual comparison, not against intra-lingual comparison.

\section{Common Orthographic Space}

Writing systems used by languages differ very widely. This can be taken to mean that there is no common orthographic space for meaningful crosslingual comparison of orthographic forms. This may be true in general, but for sets of languages using related scripts, we can assume a similar orthographic space. For example, most of the major South Asian languages use scripts derived from Brahmi. The similarity among these scripts is so much that crosslingual comparison of text is possible for various purposes such as identifying cognates without any phonetic transcription. This is in spite of the fact that the letter shapes differ so much that they are not mutually identifiable. Such similarity is relevant for corpus based measures.

\section{Corpus Based Measures}

Since we use (non-parallel) corpora of the two languages for finding out the cognates and hence comparing two languages, the validity of the results depends on how representative the corpora are. However, if they are of enough size, we might still be able to make meaningful, even if limited, comparison among languages. We restrict ourselves to word list based comparison. In such a case, cor- pus based measures can be effective if the corpora contain a representative portion of the vocabulary, or even of word segments. The second case (of segments) is relevant for the $n$-gram measure described in section-7.

This category of measures have to incorporate more linguistic information if they are to provide good results. Designing such measures can be a challenging problem as we will be mainly relying on the corpus for our information. Knowledge about similarities and differences of writing systems can play an important role here. The two cognate based measures described in sections 9 and 10 are an attempt at this. But first we describe a simple $n$-gram based measure.

\section{Symmetric Cross Entropy (SCE)}

The first measure is purely a letter $n$-gram based measure similar to the one used by Singh (Singh, 2006b) for language and encoding identification. To calculate the distance, we first prepare letter 5-gram models from the corpora of the languages to be compared. Then we combine $n$-grams of all orders and rank them according to their probability in descending order. Only the top $N n$-grams are retained and the rest are pruned. ${ }^{1}$ Now we have two probability distributions which can be compared by a measure of distributional similarity. We have used symmetric cross entropy as such a measure:

$$
d_{s c e}=\sum_{g_{l}=g_{m}}\left(p\left(g_{l}\right) \log q\left(g_{m}\right)+q\left(g_{m}\right) \log p\left(g_{l}\right)\right)
$$

where $p$ and $q$ are the probability distributions for the two languages and $g_{l}$ and $g_{m}$ are $n$-grams in languages $l$ and $m$, respectively.

The disadvantage of this measure is that it does not use any linguistic (e.g., phonetic) information, but the advantage is that it can measure the similarity of distributions of $n$-grams. Such measures have proved to be very effective in automatically identifying languages of text, with accuracies nearing $100 \%$ for fairly small amounts of training and test data (Adams and Resnik, 1997; Singh, 2006b).

\footnotetext{
${ }^{1}$ This is based on the results obtained by Cavnar (Cavnar and Trenkle, 1994) and our own studies, which show that the top $N$ (300 according to Cavnar) $n$-grams have a high correlation with the identity of the language.
} 


\section{Method for Cognate Identification}

The other two measures are based on cognates, inherited as well as borrowed. Both of them use an algorithm for identification of cognates. Many such algorithms have been proposed. Estimates of surface similarity can be used for finding cognate words across languages for related languages. By surface similarity we mean the orthographic, phonetic and (possibly) morphological similarity of two words or strings. In spite of the name, surface similarity is deeper than string similarity as calculated by edit distances. Ribeiro et al. (Ribeiro et al., 2001) have surveyed some of the algorithms for cognate alignment. However, since they studied methods based on parallel text, we cannot use them directly.

For identifying cognates, we are using the computational model of scripts or CPMS (Singh, 2006a). This model takes into account the characteristics of Brahmi origin scripts and calculates surface similarity in a fuzzy way. This is achieved by using a stepped distance function (SDF) and a dynamic programming (DP) algorithm. We have adapted the CPMS for identifying cognates.

Different researchers have argued about the importance of order sensitivity and scaling in using string comparison algorithms (Heeringa et al., 2006; Ellison and Kirby, 2006). The CPMS takes both of these into account, as well as using knowledge about the script. In general, the distance between two strings can be defined as:

$$
c_{l m}=f_{p}\left(w_{l}, w_{m}\right)
$$

where $f_{p}$ is the function which calculates surface similarity based cost between the word $w_{l}$ of language $l$ and the word $w_{m}$ of language $m$.

Those word pairs are identified as cognates which have the least cost.

\section{Cognate Coverage Distance (CCD)}

The second measure used by us is a corpus based estimate of the coverage of cognates across two languages. Cognate coverage is defined as the number of words (out of the vocabularies of the two languages) which are of the same origin. The decision about whether two words are cognates or not is made on the basis of surface similarity of the two words as described in the previous section. We use (nonparallel) corpora of the two languages for identifying the cognates.

The normalized distance between two languages is defined as:

$$
t_{l m}^{\prime}=1-\frac{t_{l m}}{\max (t)}
$$

where $t_{l m}$ and $t_{m l}$ are the number of cognates found when comparing from language $l$ to $m$ and from language $m$ to $l$, respectively.

Since the CPMS based measure of surface lexical similarity is asymmetric, we calculate the average number of unidirectional cognates:

$$
d^{c c d}=\frac{t_{l m}^{\prime}+t_{m l}^{\prime}}{2}
$$

\section{Phonetic Distance of Cognates (PDC)}

Simply finding the coverage of cognates may indicate the distance between two languages, but a measure based solely on this information does not take into account the variation between the cognates themselves. To include this variation into the estimate of distance, we use another measure based on the sum of the CPMS based cost of $n$ cognates found between two languages:

$$
C_{l m}^{p d c}=\sum_{i=0}^{n} c_{l m}
$$

where $n$ is the minimum of $t_{l m}$ for all the language pairs compared.

The normalized distance can be defined as:

$$
C_{l m}^{\prime}=\frac{C_{l m}^{p d c}}{\max \left(C^{p d c}\right)}
$$

A symmetric version of this cost is then calculated:

$$
d_{p d c}=\frac{C_{l m}^{\prime}+C_{m l}^{\prime}}{2}
$$

\section{Experimental Setup}

For synchronic comparison, we selected ten languages for our experiment (table-1), mainly because sufficient corpora were available for these languages. These languages, though belonging to two different families (Indo-Iranian and Dravidian), have 

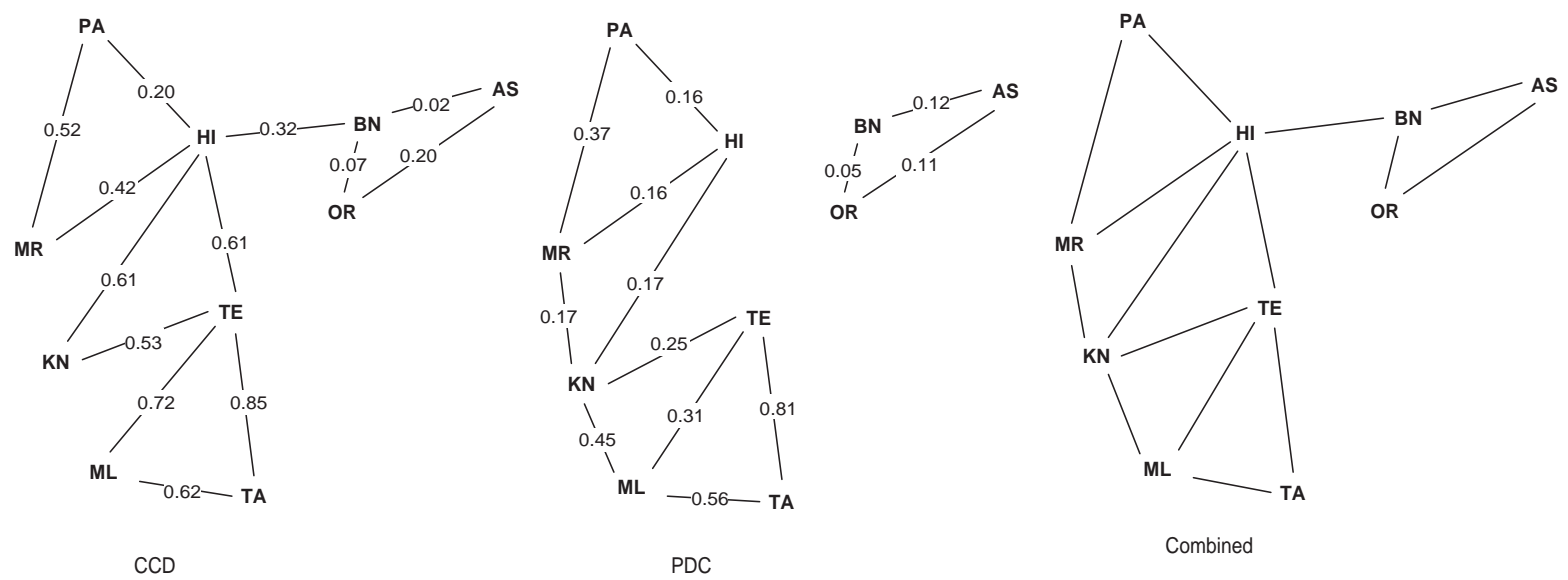

Figure 1: Graphical view of synchronic comparison among ten major South Asian languages using CCD and PDC measures. The layout of the graph is modeled on the geographical locations of these languages. The connections among the nodes of the graph are obtained by joining each node to its two closest neighbors in terms of the values obtained by using the two measures.

a lot of similarities (Emeneau, 1956). The cognate words among them are loanwords as well as inherited words. In fact, the similarity among these languages is due to common origin (intra-family) as well as contact and borrowing over thousands of years (intra- and inter-family). Moreover, they also use scripts derived from the same origin (Brahmi), which allows us to use the CPMS for identifying cognates. The corpora used for these ten languages are all part of the CIIL (Central Institute of Indian Languages) multilingual corpus. This corpus is a collection of documents from different domains and is one of best known corpora for Indian languages. Still, the representativeness of this corpus may be a matter of debate as it is not as large and diverse as the BNC (British National Corpus) corpus for English.

For the cognate measures (CCD and PDC), the only information we are extracting from the corpora are the word types and their frequencies. Thus, in a way, we are also working with word lists, but our word lists are extracted from corpora. Word lists handcrafted by linguists may be very useful, but they are not always available for all kinds of inter-language or inter-dialectal comparison, whereas electronic corpora are more likely to be available. Currently we are not doing any preprocessing or stemming on the word lists before running the cognate extraction algorithm. For SCE, $n$-gram models are being prepared as described in section7. For all three measures, we calculate the distances among all possible pairs of the languages.

For diachronic comparison, we selected modern standard Hindi, medieval Hindi (actually, Avadhi) and Sanskrit. The corpus for modern Hindi was the same as that used for synchronic comparison. The medieval Hindi we have experimented with is of two different periods. These are the varieties used by two great poets of that period, namely Jaayasi (14771542 A.D.) and Tulsidas (1532-1623 A.D.). We took some of their major works available in electronic form as the corpora. For Sanskrit, we used the electronic version of Mahabharata (compiled during the period 1000 B.C. to 500 A.D. approximately) as the corpus. We calculate the distances among all possible pairs of the four varieties using the three measures. We also compare the ten modern languages with Sanskrit using the same Mahabharata corpus.

For synchronic comparison, we first extract the list of word types with frequencies from the corpus. Then we rank them according to frequency. Top $N$ of these are retained. This is done because otherwise a lot of less relevant word types like proper nouns get included. We are interested in comparing the core vocabulary of languages. The assumption is that words in the core vocabulary are likely to be more frequent. Another reason for restricting the experiments to the top $N$ word types is that there 


\begin{tabular}{|c|c|c|c|c|c|c|c|c|c|}
\hline & $\overline{\mathbf{B N}}$ & HI & KN & ML & MR & OR & $\begin{array}{l}\mathbf{P A} \\
\end{array}$ & TA & TE \\
\hline \multirow{3}{*}{$\mathbf{A S}$} & $\overline{0.02}$ & $\overline{c 0.39}$ & $\overline{c 0.71}$ & $\overline{c 0.86}$ & 0.61 & 0.20 & 00.61 & $\overline{00.93}$ & 0.73 \\
\hline & 0.12 & 0.25 & 0.39 & 0.61 & 0.45 & 0.11 & 0.58 & 0.95 & 0.46 \\
\hline & 0.05 & 0.30 & 0.51 & 0.50 & 0.43 & 0.18 & 0.42 & 0.70 & 0.64 \\
\hline \multirow[t]{3}{*}{$\overline{\mathbf{B N}}$} & & 0.32 & 0.68 & 0.86 & 0.57 & 0.07 & 0.56 & 0.96 & 0.70 \\
\hline & & 0.29 & 0.42 & 0.64 & 0.42 & 0.05 & 0.56 & 0.90 & 0.50 \\
\hline & & 0.29 & 0.47 & 0.45 & 0.43 & 0.14 & 0.42 & 0.74 & 0.43 \\
\hline \multirow[t]{3}{*}{$\mathbf{H I}$} & & & 0.61 & 0.81 & 0.42 & 0.40 & 0.20 & 0.93 & 0.61 \\
\hline & & & 0.17 & 0.56 & 0.16 & 0.27 & 0.16 & 0.87 & 0.38 \\
\hline & & & 0.43 & 0.46 & 0.16 & 0.33 & 0.20 & 0.74 & 0.34 \\
\hline \multirow[t]{3}{*}{ KN } & & & & 0.77 & 0.68 & 0.75 & 0.73 & 0.88 & 0.53 \\
\hline & & & & 0.45 & 0.17 & 0.31 & 0.50 & 0.82 & 0.25 \\
\hline & & & & 0.18 & 0.38 & 0.52 & 0.58 & 0.42 & 0.09 \\
\hline \multirow[t]{3}{*}{$\overline{\mathbf{M L}}$} & & & & & 0.89 & 0.88 & 0.88 & 0.62 & 0.72 \\
\hline & & & & & 0.65 & 0.59 & 0.77 & 0.56 & 0.31 \\
\hline & & & & & 0.42 & 0.53 & 0.55 & 0.07 & 0.19 \\
\hline \multirow[t]{3}{*}{$\overline{\mathrm{MR}}$} & & & & & & 0.64 & 0.52 & 0.95 & 0.68 \\
\hline & & & & & & 0.40 & 0.37 & 0.94 & 0.46 \\
\hline & & & & & & 0.34 & 0.39 & 0.60 & 0.30 \\
\hline \multirow[t]{3}{*}{$\overline{\mathrm{OR}}$} & & & & & & & 0.63 & 0.98 & 0.74 \\
\hline & & & & & & & 0.45 & 0.89 & 0.44 \\
\hline & & & & & & & 0.65 & 0.83 & 0.64 \\
\hline \multirow[t]{2}{*}{$\overline{\mathbf{P A}}$} & & & & & & & & 0.90 & 0.71 \\
\hline & & & & & & & & 0.90 & 0.59 \\
\hline \multirow[t]{3}{*}{ TA } & & & & & & & & & 0.08 \\
\hline & & & & & & & & & 0.81 \\
\hline & & & & & & & & & 0.39 \\
\hline
\end{tabular}

Table 1: Inter-language comparison among ten major South Asian languages using three corpus based measures. The values have been normalized and scaled to be somewhat comparable. Each cell contains three values: by CCD, PDC and SCE.

are huge differences in sizes of corpora of different languages. In the next step we identify the cognates among these word lists. No language specific features or thresholds are used. Only common thresholds are used. We now branch out to using either CCD or PDC.

The method used for diachronic comparison is similar except that $N$ is much smaller because the amount of classical corpus being used (Jaayasi, Tulsidas) is also much smaller. Two letter codes are used for ten languages and four varieties ${ }^{2}$.

\section{Analysis of Results}

The results of our experiments are shown tables 1 to 3 and figures 1 and 2. Table- 1 shows the distances among pairs of languages using the three

\footnotetext{
${ }^{2}$ AS: Assamese, BN: Bengali, HI: Hindi, KN: Kannada, ML: Malayalam, MR: Marathi, OR: Oriya, PA: Punjabi, TA: Tamil, TE: Telugu, TL: Avadhi (Tulsidas), JY: Avadhi (Jaayasi), MB: Sanskrit (Mahabharata)
}

measures. Figure-1 shows a graph showing the distances according to CCD and PDC. Figure- 2 shows the effect of the size of word lists $(N)$ on comparison for three linguistically close language pairs. Table-2 shows the comparison of ten languages with Sanskrit. Table-3 gives the diachronic comparison among four historical varieties.

\subsection{Synchronic Comparison}

As table-1 shows, all three measures give results which correspond well to the linguistic knowledge about differences among these languages. Cognate based measures give better results, but even the $n$ gram based measure gives good results. However, there are some differences among the values obtained with different measures. These differences are also in accordance with linguistic insights. For example, the distance between Hindi and Telugu was given as 0.61 by CCD and 0.38 by PDC. Similarly, the distance between Hindi and Kannada was given as 0.61 by CCD and 0.17 by PDC. These values, in relative terms, indicate that the number of cognates between these languages is in the medium range as compared to other pairs. But less PDC cost shows that top $N$ cognates are very similar. This is because most cognates are tatsam words directly borrowed from Sanskrit without any change.

The results presented in the table have been normalized on all language pairs using the maximum and minimum cost. The results would be different and more comparable if we normalize over language families (Indo-Iranian and Dravidian). With such normalization, Punjabi-Oriya and MarathiAssamese are identified as the farthest language pairs with costs of 0.92 and 0.90 , respectively. This corresponds well with the actual geographical and linguistic distances.

While comparing with Sanskrit, it is clear that different languages have different levels of cognate coverage. However, except for Punjabi and Tamil, all languages have very similar PDC cost with the Mahabharata corpus. This again shows that the closest cognates among these languages are tatsam words. These results agree well with linguistic knowledge, even though the Sanskrit corpus (Mahabharata) is highly biased.

Figure-1 makes the results clearer. It shows that just by connecting each node to its nearest two 


\begin{tabular}{|c|c|c|c|c|c|c|c|c|c|c|}
\hline Distance & AS & BN & HI & KN & ML & MR & OR & PA & TA & TE \\
\hline CCD & 0.71 & 0.70 & 0.65 & 0.78 & 0.87 & 0.73 & 0.71 & 0.78 & 0.94 & 0.77 \\
\hline PDC & 0.37 & 0.38 & 0.40 & 0.43 & 0.37 & 0.41 & 0.37 & 0.50 & 0.63 & 0.30 \\
\hline
\end{tabular}

Table 2: Comparison with Sanskrit (Mahabharata)

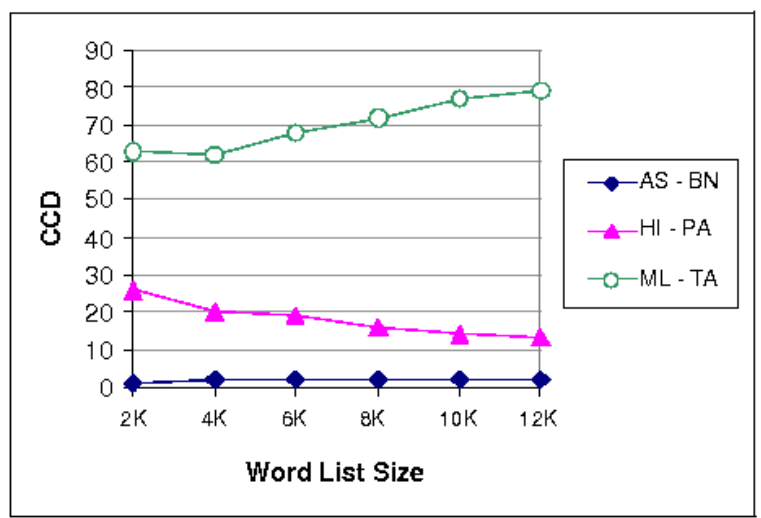

Figure 2: Effect of the size of word lists on interlanguage comparison.

\begin{tabular}{|c|ccc|}
\hline & TL & JY & MB \\
\hline \hline HI & 0.45 & 0.54 & 0.82 \\
& 0.45 & 0.42 & 0.70 \\
& 0.64 & 0.56 & 0.49 \\
\hline TL & & 0.01 & 0.84 \\
& & 0.02 & 0.72 \\
& & 0.16 & 0.91 \\
\hline JY & & & 0.98 \\
& & & 0.95 \\
& & & 0.81 \\
\hline
\end{tabular}

Table 3: Diachronic comparison among four historical varieties.

neighbors we can get a very good graphical representation of the differences among languages. It also shows that different measures capture different aspects. For example, CCD fails to connect Marathi with Kannada and Kannada with Malayalam. Similarly, PDC fails to connect Bengali with Hindi. We get this missing information by combining the graphs obtained with the two measures. More sophisticated methods for creating such graphs may give better results. Note that the Hindi-Telugu and Marathi-Kannada connections are valid as these language pairs are close, even though they are not genetically related. The results indicate closeness between two languages, but they do not distinguish be- tween inheritance and borrowing.

We also experimented with several word list sizes. In figure- 2 the $C C D$ values are plotted against word list sizes for three close language pairs. There is variation for Hindi-Punjabi and Malayalam-Telugu, but not for Assamese-Bengali. The following observations can be derived from the three lines on the plot. Malayalam-Telugu share a lot of common core words but not less common words. Hindi-Punjabi share a lot of less common words, but core words are not exactly similar. Finally, Assamese-Bengali share both core as well as less common words.

\subsection{Diachronic Comparison}

Table-4 shows the results. We can see that Hindi is closer to Tulsidas than to Jaayasi by the CCD measure. PDC gives almost similar results for both. Tulsidas and Jaayasi are the nearest. Tulsidas is much nearer to Mahabharata than Jaayasi, chiefly because Tulsidas' language has more Sanskrit origin words. Our results put Tulsidas nearest to Hindi, followed by Jaayasi and then Sanskrit. This is historically as well as linguistically correct.

\section{Conclusions and Further Work}

In this paper we first discussed the possibility and validity of using corpus based measures for comparative study of languages. We presented some arguments in favor of this possibility. We then described three corpus based measures for comparative study of languages. The first measure was symmetric cross entropy of letter $n$-grams. This measure uses the least amount of linguistic information. The second and third measures were cognate coverage distance and phonetic distance of cognates, respectively. These two are more linguistically grounded. Using these measures, we presented a synchronic comparison of ten major South Asian languages and a diachronic comparison of four historical varieties. The results of our experiments show that even these simple measures based on crosslingual comparison 
and on the data extracted from not very representative and noisy corpora can be used for obtaining or validating useful linguistic insights about language divergence, classification etc.

These measures can be tried for more languages to see whether they have any validity for less related languages than the languages we experimented with. We can also try to design measures and find methods for distinguishing between borrowed and inherited words. Proper combination of synchronic and diachronic comparison might help us in doing this. Other possible applications could be for language reconstruction, classification, dialectology etc.

Better versions of the two cognate based measures can be defined by using the idea of confusion probabilities (Ellison and Kirby, 2006) and the idea of distributional similarity. If intra-lingual comparison is more meaningful than inter-lingual comparison, then these modified versions should be even more useful for comparative study of languages.

\section{References}

Gary Adams and Philip Resnik. 1997. A language identification application built on the Java client-server platform. In Jill Burstein and Claudia Leacock, editors, From Research to Commercial Applications: Making NLP Work in Practice, pages 43-47. Association for Computational Linguistics.

William B. Cavnar and John M. Trenkle. 1994. N-grambased text categorization. In Proceedings of SDAIR94, 3rd Annual Symposium on Document Analysis and Information Retrieval, pages 161-175, Las Vegas, US.

I. Dyen, J.B. Kruskal, and P. Black. 1992. An indo-european classification: A lexicostatistical experiment. In Transactions of the American Philosophical Society, 82:1-132.

T. Mark Ellison and Simon Kirby. 2006. Measuring language divergence by intra-lexical comparison. In Proceedings of the 21 st International Conference on Computational Linguistics and 44th Annual Meeting of the Association for Computational Linguistics, Sydney, Australia. Association for Computational Linguistics.

M. B. Emeneau. 1956. India as a linguistic area. In Linguistics 32:3-16.

W. Heeringa, P. Kleiweg, C. Gooskens, and J. Nerbonne. 2006. Evaluation of String Distance Algorithms for Dialectology. In Proc. of ACL Workshop on Linguistic Distances.
G. Kondrak and T. Sherif. 2006. Evaluation of Several Phonetic Similarity Algorithms on the Task of Cognate Identification. In Proc. of ACL Workshop on Linguistic Distances.

Grzegorz Kondrak. 2002. Algorithms for language reconstruction. Ph.D. thesis. Adviser-Graeme Hirst.

April McMahon and Robert McMahon. 2005. Language Classification by the Numbers. Oxford University Press, Oxford.

Luay Nakleh, Don Ringe, and Tandy Warnow. 2005. Perfect phylogentic networks: A new methodology for reconstructing the evolutionary history of natural languages. pages 81-2:382-420.

J. Nerbonne and W. Heeringa. 1997. Measuring dialect distance phonetically. In Proceedings of SIGPHON97: 3rd Meeting of the ACL Special Interest Group in Computational Phonology.

J. Nerbonne. 2005. Review of 'language classification by the numbers' by april mcmahon and robert mcmahon.

B. Port and A. Leary. 2005. Against formal phonology. pages 81(4):927-964.

M. Remmel. 1980. Computers in the historical phonetics and phonology of Balto-Finnic languages: problems and perspectives. In Communication présentée au 5 th International Finno-Ugric Congress, Turku.

A. Ribeiro, G. Dias, G. Lopes, and J. Mexia. 2001. Cognates alignment. Machine Translation Summit VIII, Machine Translation in The Information Age, pages 287-292.

Duan sheng Chen and Zheng kai Liu. 2003. A novel approach to detect and correct highlighted face region in color image. In AVSS '03: Proceedings of the IEEE Conference on Advanced Video and Signal Based Surveillance, page 7, Washington, DC, USA. IEEE Computer Society.

Anil Kumar Singh. 2006a. A computational phonetic model for indian language scripts. In Constraints on Spelling Changes: Fifth International Workshop on Writing Systems, Nijmegen, The Netherlands.

Anil Kumar Singh. 2006b. Study of some distance measures for language and encoding identification. In Proceedings of ACL 2006 Workshop on Linguistic Distance, Sydney, Australia.

M. Swadesh. 1952. Lexico-dating of prehistoric ethnic contacts. In Proceedings of the American philosophical society, 96(4). 